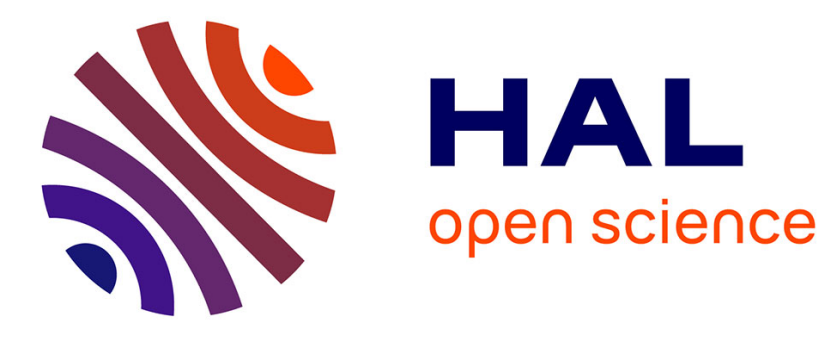

\title{
Adhesion of soft objects on wet substrates
}

Françoise Brochard-Wyart, Axel Buguin, Pascal Martin, Arnaud Martin,

Olivier Sandre

\section{To cite this version:}

Françoise Brochard-Wyart, Axel Buguin, Pascal Martin, Arnaud Martin, Olivier Sandre. Adhesion of soft objects on wet substrates. Journal of Physics: Condensed Matter, 2000, 12 (8A), pp.A239-A244. 10.1088/0953-8984/12/8A/330 . hal-02168817

\section{HAL Id: hal-02168817 https://hal.science/hal-02168817}

Submitted on 8 Oct 2019

HAL is a multi-disciplinary open access archive for the deposit and dissemination of scientific research documents, whether they are published or not. The documents may come from teaching and research institutions in France or abroad, or from public or private research centers.
L'archive ouverte pluridisciplinaire HAL, est destinée au dépôt et à la diffusion de documents scientifiques de niveau recherche, publiés ou non, émanant des établissements d'enseignement et de recherche français ou étrangers, des laboratoires publics ou privés. 


\title{
Adhesion of soft objects on wet substrates
}

\author{
F Brochard-Wyart, A Buguin, P Martin, A Martin and O Sandre \\ Laboratoire Physico-Chimie Curie, UMR 168, Institut Curie, 11, rue Pierre et Marie Curie, \\ 75231 Paris, Cédex 05, France \\ E-mail: brochard@curie.fr
}

Received 15 September 1999

\begin{abstract}
We study the dynamics of contact of a soft object (rubber bead, soft shell, vesicles, living cells) on a wet substrate by removal of the intercalated liquid film. The profiles of the contact zone are observed by reflection interference contrast microscopy. The adhesion forces (either hydrophobic, electrostatic or specific) are measured by micropipettes, flow cells or 'microkarcher' techniques. For vesicles, the adhesion induces a tension of the membrane, which relaxes by the formation of transient macroscopic pores. We study the dynamics of opening and closing of pores.
\end{abstract}

\section{Introduction}

We study how soft deformable objects adhere on a wet substrate by elimination of the water intercalated liquid film. Stability of intercalated liquid film is of crucial interest for many practical applications. One example is the irreversible rupture of the lachrymal film, which has been observed with silicone contact lenses: the elastomer adheres strongly to the cornea and can cause severe damage when it is removed. On the contrary, when driving on a wet road, we require the water film to be squeezed away - in order to maximize grip-during the time (typically $5 \mathrm{~ms}$ ) when the tyre is exposed to water. In a similar way, a living cell which comes to adhere on a substrate must eliminate the intercalated water film. The removal of the intercalated liquid film proceeds in two steps: first drainage, then dewetting of the film by nucleation and growth of a dry contact between the solid and the soft material. This dewetting process, which controls the dynamics of adhesive contact, has not been discussed for biological systems. On the other hand, the active processes of cytoskeleton reorganization and the characterization and organization of specific molecules responsible for cell adhesion are now well understood.

We have selected three systems to study the growth of dry contact of a soft object adhering on a wet model substrate:

(1) latex spheres;

(2) vesicles and

(3) mushroom spores.

These systems will allow us to study systematically the time required to achieve adhesion. The key parameters are the volume or surface rigidity of the deformable object, and the nature of adhesive bonds (hydrophobic, electrostatic or steric). 


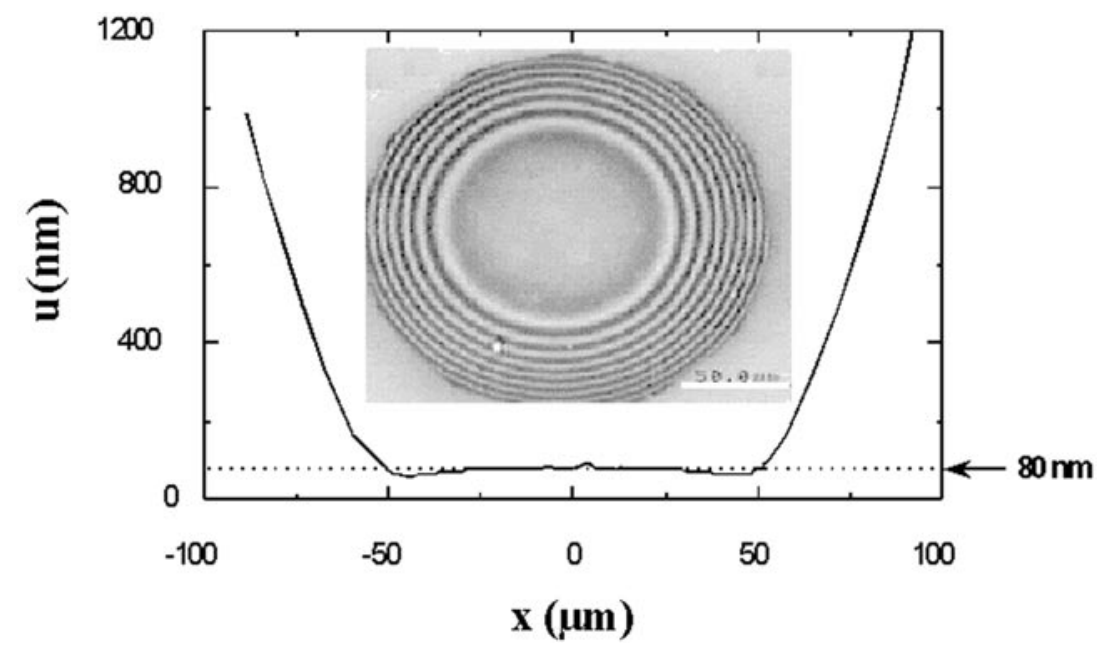

(a)
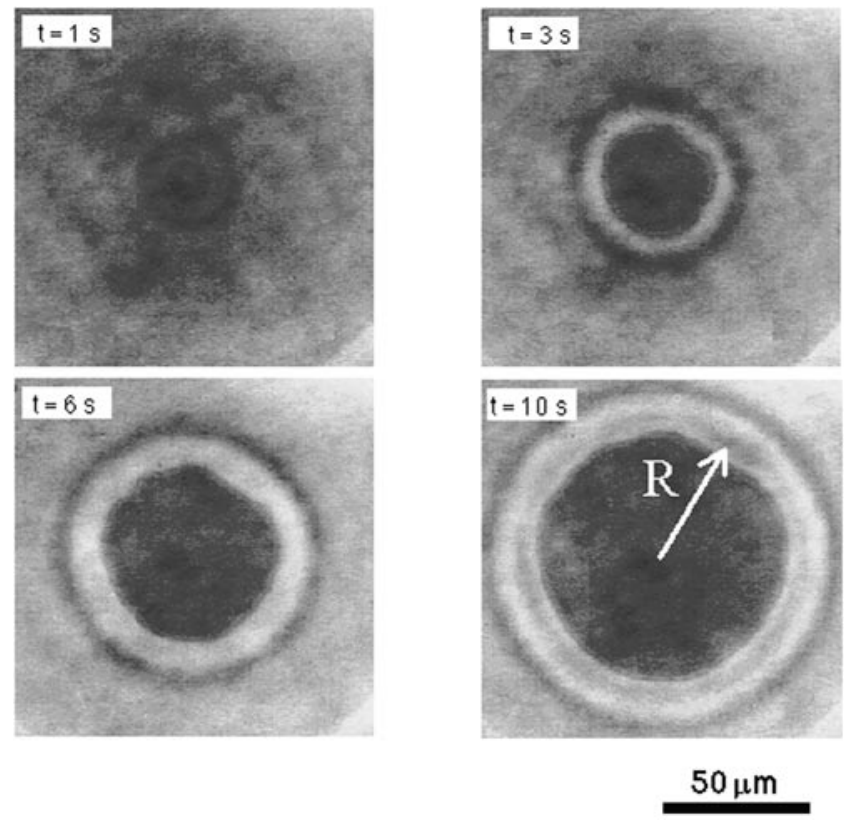

(b)

Figure 1. Dewetting at soft interface: (a) profile of an intercalated liquid film sandwiched between a rigid solid (a silanized glass plate) and a PDMS rubber (P Martin) obtained by reflection interference contrast microscopy (RICM); (b) nucleation and growth of a dry solid/rubber contact nucleated through an intercalated liquid film (viscosity $\eta=2.65 \mathrm{~Pa} \mathrm{~s}$, thickness $e=130 \mathrm{~nm}$ ). On the first image, we can notice the presence of the defect at the centre of the film used to nucleate the dry contact. A flat rim surrounding the dry patch is clearly visible (P Martin).

\section{Latex beads}

We use a polydimethylsiloxane rubber cap prepared by reticulation of droplets of reactive mixture. They are optically smooth and they behave like a pure elastic medium (elastic modulus 

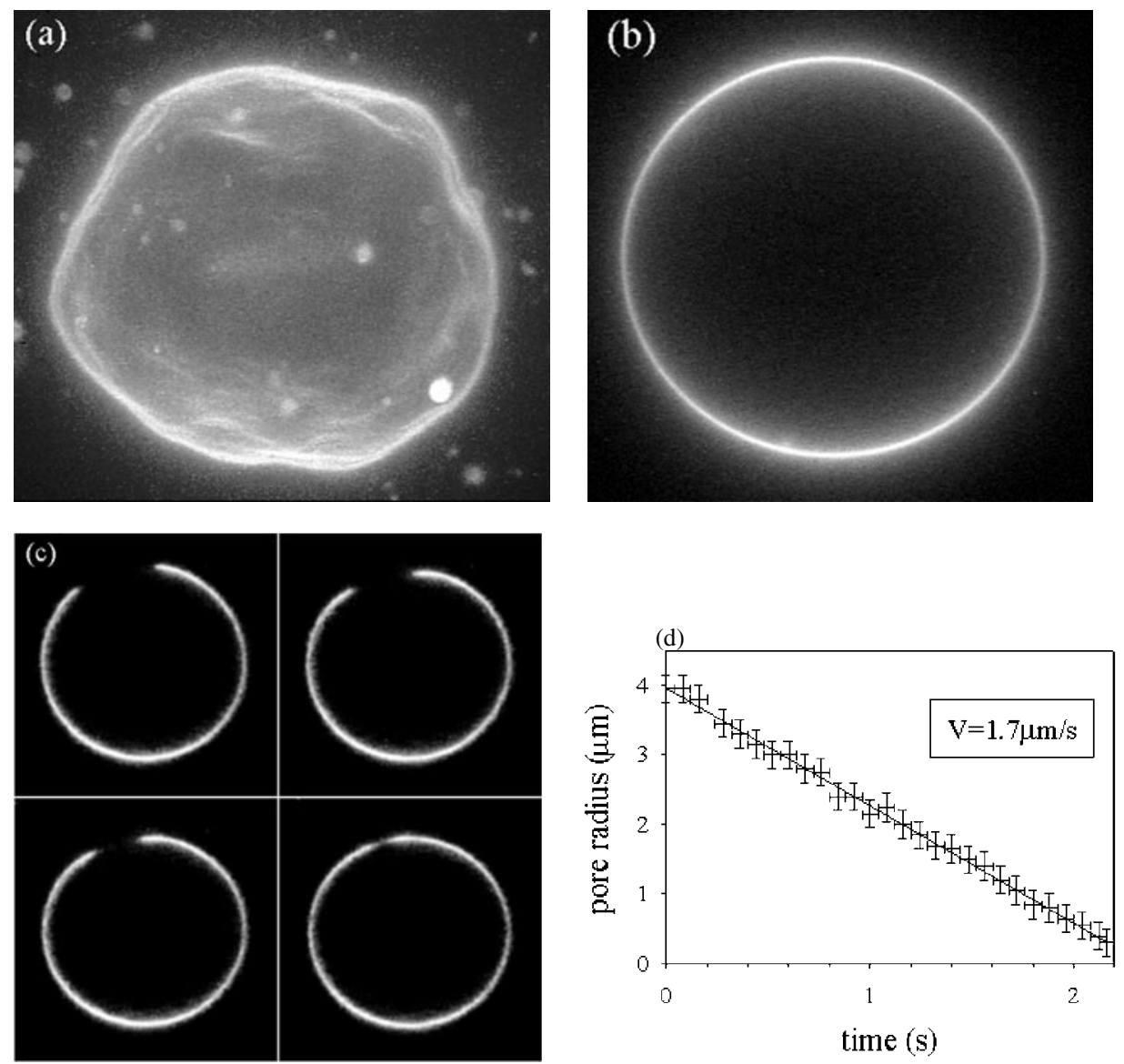

Figure 2. Adhesion of giant vesicles. (a) Undulations by thermal fluctuation of a free vesicle. The surface tension is zero and the surface is crumpled. (b) Tense spherical vesicle: a weak adhesion absorbs excess surface and the membrane has a surface tension. (c) Transient pores in stretched vesicles. Addition of glycerol raises the viscosity up to 30 cpoise and slows down the leaking of the inner liquid, which enables us to observe macroscopic transient pores (O Sandre). (d) Closure of a pore at constant velocity.

$\mu=10^{5} \mathrm{~Pa}$ ). Intercalated films (thickness $e$ ) are formed by elastic indentation of the rubber cap pressed against a hydrophobic glass plate through a separating liquid drop (figure 1(a)). The stability of these films is controlled by the spreading coefficient $S=\gamma_{\mathrm{SR}}-\left(\gamma_{\mathrm{SL}}+\gamma_{\mathrm{LR}}\right)$. In our case, $S$ is negative: the system gains energy by excluding the intercalated liquid. We have measured $S$ from the equilibrium profile of sessile droplets standing at the solid/rubber interface. These droplets are flat semi-ellipsoid, with thickness $H(\approx \mu \mathrm{m})$ related to their diameter $D(\approx 100 \mu \mathrm{m})$ by [1] $H^{2} \cong h_{0} D$ where $h_{0}=|S| / \mu$ defines a characteristic length. We find $h_{0}=640 \AA$ for water, leading to $S=-50 \mathrm{mN} \mathrm{m}^{-1}$.

The intercalated films are metastable, and dewet by nucleation and growth of a dry contact. Only microscopic films ( $e \approx 100 \mathrm{~nm}$ ) dewet, because the radius $R_{\mathrm{C}}$ to nucleate a dry contact $\left(R_{\mathrm{C}} \approx e^{2} / h_{0}\right.$ ) becomes huge for thicker films. We have observed by optical interferometry the radius of one single dry contact (figure 1(b)) expanding with time, and the profile of the 
surrounding rim. We find that [2] (i) the shape of the rim squeezed by the rubber is quasi-static, and (ii) the radius $R(t)$ of the contact increases with time according to an unusual power law $R(t) \approx t^{3 / 4}$. These results are interpreted in terms of a hydrodynamic model [3], assuming viscous dissipation in the moving rim while the rubber is purely elastic.

\section{Vesicles}

We have also studied the adhesion of giant vesicles of phospholipids, produced by electroformation [4]. The vesicles are labelled with a lipophilic dye and imaged by fluorescence microscopy. The glass slides of the observation chamber are bare or treated with an adhesive coating for the vesicles. Incubated with a glucose solution containing $\mathrm{CaCl}_{2}, \mathrm{Ca}^{2+}$ adsorbs strongly to the phosphatidylcholine head groups of the bilayer, thus conferring to the vesicles a net positive charge. Therefore, they are attracted on the negative surface of bare glass at neutral $\mathrm{pH}$. The second method is to adsorb polycationic chains onto the glass substrates. The intensity of the electrostatic interaction can be modulated by the calcium concentration in the former case, and by the $\mathrm{pH}$ in the second case.

The free vesicles fluctuate (figure 2(a)), because they have a zero surface tension. When they adhere on the substrate, they become stretched and spherical (figure 2(b)). The contact formation of the vesicle with the substrate is observed by RICM (figure 3). The full image is achieved with a fluorescence microscope.

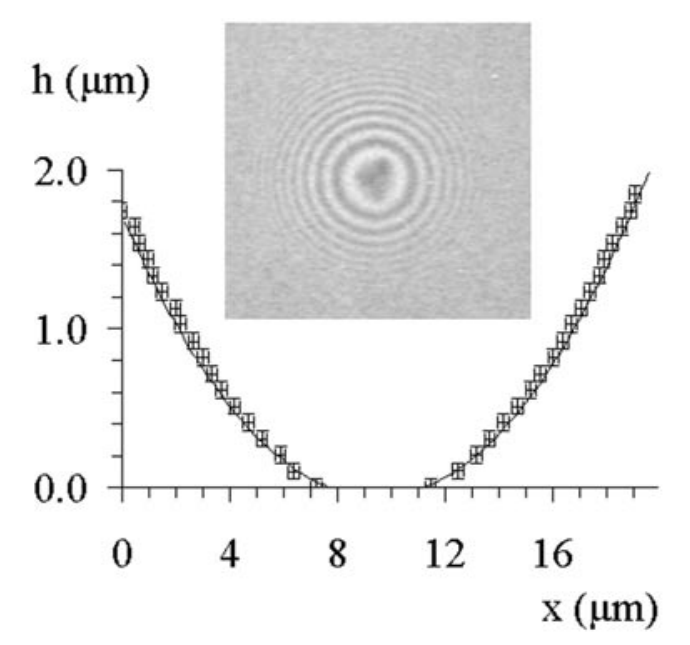

Figure 3. Reconstruction by RICM of a giant vesicle profile, which adheres weakly on a charged substrate (O Sandre).

We observe macroscopic transient pores (figure 2(c)) in tense vesicles. Holes open above a critical radius $r_{\mathrm{cl}}$, grow to a radius $r_{\mathrm{c} 2}$ and close back. We interpret the upper limit by a relaxation of the membrane tension as the holes expand. The closing of the holes (figure 2(d)) is due to a further relaxation of the surface tension when the internal liquid leaks out. We can slow down the leak-out kinetics by increasing the viscosity of the ambient solution. The radius of the transient pores becomes very large, up to $10 \mu \mathrm{m}$. A dynamical model fits our data for the growth and closure of pores [5].

The membrane permeability can be modulated by the adhesion strength. This may have important application for biological processes, such as exocytosis or endocytosis. 

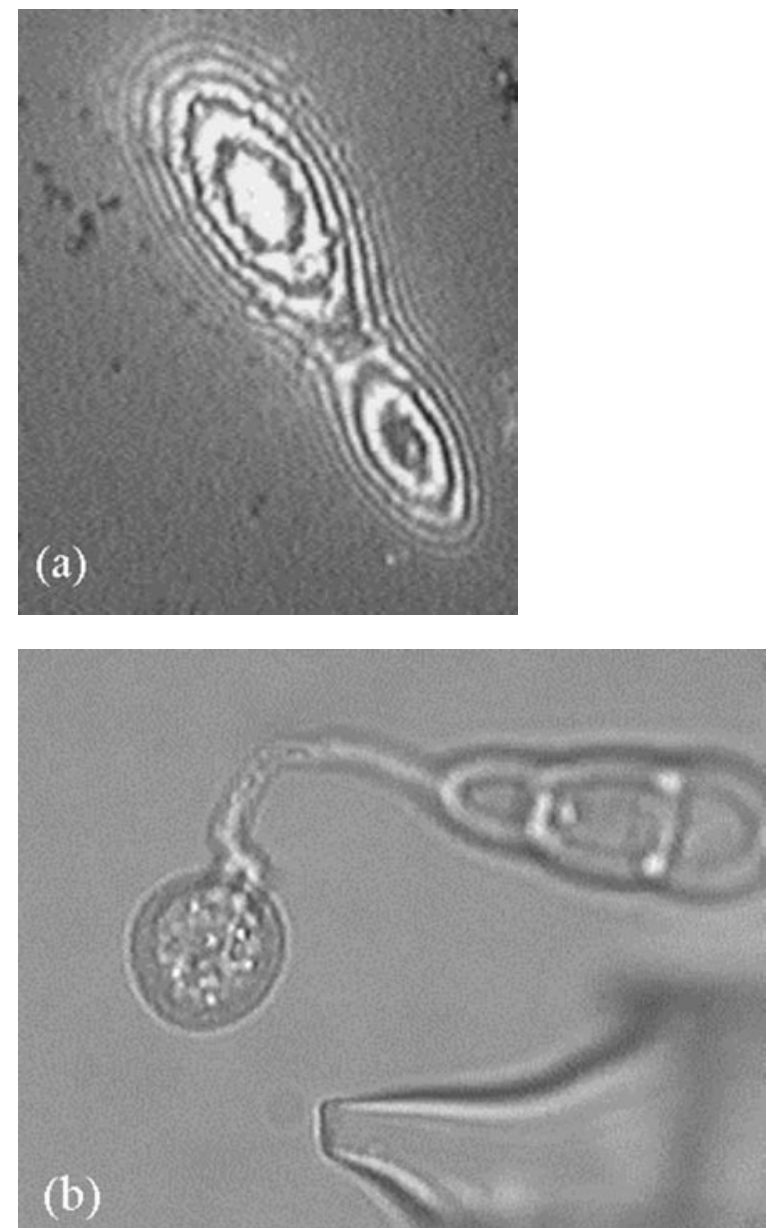

Figure 4. Adhesion of a spore of Piricularia orizae (A Buguin). (a) After one minute of incubation, a spore starts to adhere. (b) After a few hours, the development is achieved: spore (in the shape of a pear), germination tube and appressorium.

In the near future, we want to study (1) the growth of one single contact of a vesicle pressed on the substrate by magnetic or optical tweezers (in this way, we can squeeze a thin film and decouple the drainage from the dewetting) and (2) the case of a reticulated membrane 'soft shell'. This intermediate case will better model living cells, where a two dimensional network of proteins is often connected to the fluid membrane.

\section{Living cells: spores of mushrooms}

The adhesion of spores (Piricularia orizae) on wet leaves controls its development and the infection of rice plantations. We study the adhesion of the spores on fluorinated glass, a substrate with a degree of hydrophobicity similar to the leaves. We show in figures 4(a) and (b) a spore after one minute and a few hours of incubation: the spore emits a 'germination tube' of length $50 \mu \mathrm{m}$ terminated by a spherical capsule of size $10 \mu \mathrm{m}$ called the 'appressorium'. This appressorium, which contains the genetic material, is a real chemical boring-machine: 
it produces small sugar molecules, which increase osmotically the inside pressure up to fifty bars to make a hole in rigid sheet, such as leaves or plastic, to inject its contents. Under shear flows, in a Hele-Shaw cell, the spores are pulled out only in the first stage of development: the adhesion energy is too strong! Micropipettes have been used to extract the spores individually and to quantify their adhesion. Increasing their diameter, it is also possible to apply an intense jet stream $\left(\approx \mathrm{m} \mathrm{s}^{-1}\right)$ able to unstick this strongly adhesive organism and to determine with accuracy the adhesion energy.

A challenge is now to understand at a molecular level the mechanism of strong adhesion on substrate more hydrophobic than Teflon and immersed in water.

\section{Acknowledgments}

We thank J M Vacher, L Vovelle, S Deroo, M.P Latorse and P G de Gennes for stimulating discussions.

\section{References}

[1] Martin P, Silberzan P and Brochard-Wyart F 1997 Langmuir 134910

[2] Martin P and Brochard-Wyart F 1998 Phys. Rev. Lett. 803296

[3] Brochard-Wyart F and de Gennes P G 1994 J. Phys.: Condens. Matter 6 A9

[4] Angelova M I, Soleau S, Mileard P, Faucon J F and Bothorel P 1992 Prog. Colloid Polym. Sci. 89127

[5] Sandre O, Moreaux L and Brochard-Wyart F 1999 Proc. Natl Acad. Sci. USA 9610591 